\title{
Optimization Model for Residential Tiered Electricity Pricing Based on Peak-Valley TOU Price
}

\author{
L. Yang, K.T. Chen, Z.F. Tan \\ School of Economics and Management \\ North China Electric Power University \\ Changping District, Beijing, China
}

\author{
B.B. Zhou \\ Liaoning Province Power Company \\ Ningbo Road, Shenyang, Liaoning, China
}

\begin{abstract}
In recent years, with the shortage of energy supply and tremendous of environmental pressure in China, the price of primary energy such as coal continues to rise, as well as the electricity price. However, the adjustable amplitude and frequency for residential electricity are lower than other industries. In order to construct resource conserving and environment friendly society, to gradually reduce the price of cross-subsidization and guide residents to save electricity, it is necessary to implement the residential tiered electricity pricing. This paper optimizes the model of residential tiered electricity pricing through the introduction of peak-valley TOU price, which lays the foundation for promotion of power system reform.
\end{abstract}

Keywords-Residential electricity price; tiered electricity pricing; peak-valley TOU price; model

\section{INTRODUCTION}

Electric power industry is an important basic industry of the national economy, and is also an important part of social public utilities. After years of efforts, China's power system reform has achieved initial results. The price reform is one of the core content of electricity market reform. Throughout the reform system, the tariff is an important economic lever. It not only can optimize the resources allocation, but also can adjust the relations among various interests [1, 2].

Residential electricity consumption is an extremely important part for electricity sales market. The establishment of residential electricity price involves thousands of families, and is related to vital interests and living standards of the masses, as well as social efficiency, effectiveness and fairness [3]. Therefore, it is significant to establish a scientific and reasonable price system for the residents. This paper introduces peak-valley TOU price to design and optimize the model of residential tiered electricity pricing, and then obtains the optimization model for operation cost of the system.

\section{RESEARCH STATUS FOR RESIDENTIAL TIERED ELECTRICITY PRICING}

In China, the tiered pricing should be described to be the increasing price by ladder. It is refers to replace the current residential price of a single form with the approach of segmented pricing according to residential consumption, in this pricing method, the price of electricity increases gradually in a stepwise form along with the growth of electricity consumption $[4,5]$. The tiered electricity price divide power level of residential consumption in accordance with three stages of "meet the basic demand", "meet the normal and reasonable demand" and "meet the high quality electricity demand". Then, the corresponding price is set up. The tariff implements principle of stepping increment [6].

Although the existing tiered electricity pricing system has been promoted and has achieved the electricity saving benefit, this system has not considered the distribution of load and lacked reaction for electricity value in peak or valley period. Actually, electric power often needs more standby unit for power generation to meet the residents' consumption in the peak period, so the generation cost is high. Additionally, the load demand is low in valley period, so many units have not been called and utilization efficiency of units is not high. If we can guide residents to use electricity in the valley time, the reducing peak and filling valley will be achieved, and the utilization efficiency of units will be improved [7, 8]. Therefore, this paper introduces the peak-valley TOU price in residential tiered electricity price, and then optimizes the sub-file number, sub-file power and block-tariff.

\section{Design Model for Residential Tiered ELECTRICITY PRICING}

To guide residents using electricity rationally, tiered electricity pricing has been introduced. The upper tiered electricity pricing optimization model is constructed to differ from the traditional concept, targeting as the minimal electricity cost for residents. Optimization cycle is $24 \mathrm{~h}$ in this paper in order to combine the tiered electricity pricing with the peak-valley price. Specific goal function is as follows.

$$
\min C=\sum_{t=1}^{T}\left\{P_{1, t} \times Q_{1, t}+\sum_{i=1}^{I}\left[P_{2 i, t} \times\left(Q_{2 i, t}-Q_{2 i-1, t}\right)+P_{2 i, t, t} \times\left(Q_{2 i+1, t}-Q_{2 i, t}\right)\right]\right\}(1)
$$

In this formula, $P_{1, t}$ refers to electricity price for the first-file resident at t time. $Q_{1, t}$ is electricity consumption for the first-file resident at t time. $P_{i, t}$ refers to electricity price for i-file resident at t time. $Q_{i, t}$ is electricity consumption for $\mathrm{i}$-file resident at $\mathrm{t}$ time.

Introducing tiered electricity price should consider the psychological strategy for residents. For the goal of reducing electricity costs and consumption, electricity costs of some users would rise after implementing tiered electricity price. In order to facilitate the expression, the comparison function $F\left(x_{1}, x_{2}\right)$ is defined.

$$
F\left(x_{1}, x_{2}\right)= \begin{cases}1, & x_{1}>x_{2} \\ 0, & x_{1} \leq x\end{cases}
$$

(1) Electricity cost constraint 
Electricity costs of some users should remain unchanged after implementing tiered electricity price. If total resident electricity is $\mathrm{m}$, then:

$$
\frac{\sum_{i=1}^{I} F\left(\tilde{C}_{i}, C_{i}\right)}{I} \geq \lambda_{1}
$$

In this formula, $\tilde{C}_{i}$ and $C_{i}$ are electricity costs before and after introducing tiered electricity price for the resident i. $\lambda_{1}$ is proportional coefficient which refers to the proportion of residents whose electricity cost is not higher than the cost before tiered electricity price.

$$
\begin{gathered}
\tilde{C}_{i}=\tilde{P}_{i} \times \tilde{Q}_{i} \\
C_{i}=\sum_{j=1}^{J} P_{i j} \times Q_{i j}
\end{gathered}
$$

In formulas above, $\tilde{P}_{i}$ and $\tilde{Q}_{i}$ refer to electricity cost and consumption before introducing tiered electricity price for the resident i. $P_{i j}$ and $Q_{i j}$ refer to electricity cost and consumption after introducing tiered electricity price for the resident $\mathrm{i}$ in $\mathrm{j}$-file.

At the same time, to ensure the implementation of tiered electricity price, electricity consumption for a certain percentage of residents should cross at least two intervals after implementing tiered electricity price, then:

$$
\frac{\sum_{i=1}^{I} F\left(Q_{j}, Q_{1}\right)}{I} \geq \lambda_{2}
$$

In this formula, $\lambda_{2}$ is the proportion of residents whose electricity consumptions have crossed at least two intervals.

To reduce residential reaction degree after introducing tiered electricity price, the basic electricity demand of residents should be limited, it is as follows.

$$
Q_{i 1} \geq \underline{Q}
$$

$\underline{Q}$ is the basic electricity demands of residents.

After determining the number of sub-grade, the block-tariff $P_{i j}^{*}$ and block-electricity $Q_{i j}^{*}$ can be obtained by solving the model above.

\section{Design Model for Peak-Valley tou Price}

Further, TOU price is introduced to reflect the time value of electricity. The main psychological factor of residents to respond TOU price is to minimize electricity costs. Therefore, residential peak-valley TOU price model is constructed as the goal of minimal electricity costs. The target function is as follows:

$$
\min C=\sum_{t=1}^{T} \sum_{i=1}^{I} P_{i t} Q_{i t}
$$

In this formula, $P_{i t}$ and $Q_{i t}$ are tariff and electricity consumption for resident $\mathrm{i}$ at $\mathrm{t}$ moment after introducing TOU price.
In order to ensure the load shifting effect of peak-valley TOU price, a certain proportion of residential electricity peak-valley difference should be decreased, the specific constraint is that:

$$
\frac{\sum_{i=1}^{m} F\left[\left(\tilde{Q}_{i}^{\max }-\tilde{Q}_{i}^{\min }\right),\left(Q_{i}^{\max }-Q_{i}^{\text {min }}\right)\right]}{I} \geq \lambda_{3}
$$

In this formula, $\tilde{Q}_{i}^{\max }$ and $Q_{i}^{\max }$ are maximum load for residents in peak period before and after TOU price. $\tilde{Q}_{i}^{\min }$ and $Q_{i}^{\min }$ are minimum load for residents in valley period before and after TOU price. $\lambda_{3}$ is residential proportion who produce the load shifting effect.

$$
\begin{aligned}
& \tilde{P}_{i}^{p} / P_{i}^{p}<1 \\
& \tilde{P}_{i}^{v} / P_{i}^{v} \geq 1
\end{aligned}
$$

In the formulas, $\tilde{P}_{i}^{p}$ and $P_{i}^{p}$ are tariff for resident $\mathrm{i}$ in peak period before and after using TOU price. $\tilde{P}_{i}^{v}$ and $P_{i}^{v}$ are tariff for resident $\mathrm{i}$ in valley period before and after using TOU price.

Considering different price characteristics of timesharing and multi-level in peak-valley step price, a concept of elastic coefficient with peak-valley and multi-level has put forward. For each resident, the tiered-TOU price in each level corresponds to an elasticity coefficient, so electricity consumption of the resident in each level is determined by peak-valley elasticity of multi-level, which can be described as follows.

$$
\left\{\begin{array}{l}
\left(\Delta Q_{i}^{p} / \tilde{Q}_{i}^{p}\right)=e_{i p}\left(\Delta Q_{i}^{p} / \tilde{Q}_{i}^{p}\right) \\
\left(\Delta Q_{i}^{v} / \tilde{Q}_{i}^{v}\right)=e_{i v}\left(\Delta Q_{i}^{v} / \tilde{Q}_{i}^{v}\right)
\end{array}\right.
$$

In this formula, $\tilde{Q}_{i j p}$ and $\tilde{Q}_{i j v}$ are electricity for resident i at $\mathrm{j}$ level in peak and valley periods when implementing a single price $\left(\tilde{Q}_{i j p}=\tilde{Q}_{i j v}\right)$. After introducing peak-valley step price, change amounts of electricity for resident $i$ at $j$ level in peak and valley periods are that: $\Delta Q_{i j p}=Q_{i j p}-\tilde{Q}_{i j p}$, $\Delta Q_{i j v}=Q_{i j v}-\tilde{Q}_{i j v} \cdot e_{i j}$ is the demand elasticity for resident $\mathrm{i}$ at $\mathrm{j}$ level.

Solving the model above, we can get optimal tariffs and electricity consumptions in peak and valley periods. Combined with the tiered price in section 2, the residential peak-valley step price can be formed.

\section{V.Optimization Model for Peak-Valley Step PRice}

The implementation of residential peak-valley step price will change electrical behavior and transfer using times of residents, which will directly affect the operation cost of the system. In order to ensure the stable operation of the system, the basic benefits of power system should be guaranteed. Therefore, an optimization model for peak-valley step price is constructed as the goal of maximizing system operation efficiency. The goal function is as follows. 


$$
\max \pi=\sum_{t=1}^{T}\left(R_{t}-\tilde{R}_{t}\right)=\sum_{t=1}^{T}\left[\sum_{i=1}^{m} C_{i t}-\sum_{i=1}^{m} c_{s t} Q_{i t}-\left(\sum_{i=1}^{m} \tilde{C}_{i t}-\sum_{i=1}^{m} c_{s t} \tilde{Q}_{i t}\right)\right]
$$

In this formula, $R_{t}$ and $\tilde{R}_{t}$ are system profits before and after implementing peak-valley step price. $c_{s t}$ refers to power purchase price for power company.

In order to optimize the system operation, system power efficiency should be promoted when reducing residential electricity cost. Specific constraints are as follows.

Firstly, changes of electricity sales profits for power companies are in a certain range. That is:

$$
\left|\frac{R-\tilde{R}}{\tilde{R}}\right| \leq \lambda_{5}
$$

Secondly, electricity sales profits should be higher than system operating costs $C_{o p}$ after implementing peak-valley step price:

$$
\sum_{i=1}^{m} C_{i} \geq C_{o p}
$$

Lastly, change magnitude of the total average price should be in a certain range:

$$
\tilde{P}-\xi_{1} \leq \frac{\sum_{i=1}^{I} C_{i}}{\sum_{i=1}^{I} Q_{i}} \leq \tilde{P}-\xi_{2}
$$

In this formula, $\xi_{1}$ and $\xi_{2}$ are constants.

Although the objective function is to reduce power consumption, electricity should satisfy the following formula after the introduction of peak-valley step price in order to improve utilization rates of existing power generating equipment.

$$
k Q_{\text {gen }}<\sum_{i=1}^{I} Q_{i}<Q_{g e n}
$$

In this formula, $Q_{g e n}$ refers to generation capacity. $k(0<k<1)$ is a scaling factor to indicate a minimum utilization of power generation equipment.

The characteristic of peak-valley step price determines the following relationship among tariffs:

$$
\left\{\begin{array}{l}
0<P_{i}^{p}<P_{i+1}^{p} \leq P_{i}^{\max }, i=1,2, \ldots, N-1 \\
0<P_{i}^{v}<P_{i+1}^{v} \leq P_{i}^{\max }, i=1,2, \ldots, N-1
\end{array}\right.
$$

In this formula, the upper limit of tariff $P$ is decided by electricity consumption level, policy, economy and other factors, which results in some differences.

\section{CONCLUSIONS}

In order to reflect the time value of electricity, this paper introduces peak-valley TOU price under the traditional residential tiered electricity price model, so peak-valley TOU price model is constructed. While the promotion of residential peak-valley TOU price directly influences the system operation cost, in order to ensure stable operation of the system, this paper constructs the optimization model for peak-valley step price with the goal of maximizing system operation benefit.

Optimization for residential tiered electricity price plays an important role in promoting the system to consume the random power resources. The final optimized model can promote random power resources into the grid, so the power output of thermal power unit will be reduced, as well as the cost of power generation system. Finally, the significant benefits would be created in economy and environment.

\section{REFERENCES}

[1] Wu Jianhong. Pricing Model of Tiered Electricity Pricing for Residents and Policy Simulation Based on Social Equilibrium [D]. North China Electric Power University, 2013, 4-7.

[2] National Development and Reform Commission improves residential tiered electricity price. National Energy Administration, http://www.nea.gov.cn/2013-12/26/c_132998400.htm

[3] Wang Ruichun. Analysis of different pricing scheme under the guidance of residential tiered electricity price $[\mathrm{J}]$. Water Resources and Power, 2013, 31(1), 215-218.

[4] Chen Minjun, Ci Xiangyang, Ping Zongfei. Residents Ladder Electricity Price Effect Evaluation Based on Analytic Hierarchy Process [J]. Science Technology and Industry, 2014, 14(9), 88-91.

[5] Li Gen. Implementation Status and Improvement Strategy of China's Residents Ladder Price[J]. Information and Communication, 2013, (2), 271-272.

[6] ZENG Ming, LI Na, LIU Chao. Energy-Saving Assessment of Tiered Pricing Program for Residential Electricity Based on Utility Function [J] East China Electric Power, 2011, 39 (8), 1215-1219.

[7] HUANG Hai-tao. Design Model for Residential Block Electricity Pricing Structure and Level [J]. East China Electric Power, 2012, 40 (5), 0721-0727.

[8] ZENG Ming. Assessment of Tiered Pricing Program for Residential Electricity Based on the Multiple Indexes [J]. East China Electric Power, 2012, 40 (3), 0359-0362. 